CPU

Revista de Investigación Educativa 18

enero-junio, 2014 | ISSN 1870-5308 | Xalapa, Veracruz

Instituto de Investigaciones en Educación | Universidad Veracruzana

\title{
Funciones y ámbitos de los asesores académicos para la diversidad lingüística y cultural
}

\author{
Dra. María Guadalupe Tinajero Villavicencio \\ Investigadora, docente \\ Instituto de Investigación y Desarrollo Educativo \\ Universidad Autónoma de Baja California, México \\ gpetinajero@gmail.com
}

El Asesor Académico para la Diversidad es un actor clave para otorgar un nuevo significado a las prácticas de los docentes del medio indígena. Desde 2003, la Secretaría de Educación Pública norma y regula las funciones que desempeñan los asesores, quienes, entre otras cosas, deben apoyar al profesorado en tres aspectos: planeación didáctica con pertinencia lingüística y cultural; incorporación de elementos a partir del contexto cultural y lingüístico local, y uso social de la lengua indígena como medio de comunicación. Este artículo presenta un análisis de los informes de seguimiento presentados en 2011 por nueve asesores de Baja California. El objetivo fue conocer las funciones que los asesores desarrollan en los centros escolares, para evidenciar su correspondencia con las estipuladas en la normativa oficial. Se puede señalar que los asesores apoyan a los docentes en dos aspectos: planeación didáctica con pertinencia lingüística y cultural, y fortalecimiento de la didáctica de enseñanza de las matemáticas, el español y las ciencias.

Palabras clave: Asesoramiento académico, educación intercultural, educación básica, diversidad cultural y lingüística. 
The Academic Advisor for Diversity is a key player who provides advisory support to teachers in indigenous school environments. In 2003, the Ministry of Education, which is responsible for regulating the role of the advisors, established three areas of support to teachers: educational planning with linguistic and cultural relevance, incorporating elements from the local cultural and linguistic context, and social use of the indigenous language as a means of communication. This paper presents an analysis of the official reports submitted to the Ministry by nine Academic Advisors for Diversity in Baja California in 2011. Our purpose is to characterize the activities that the advisors perform in schools and demonstrate their correspondence, if any, with the stipulated ministry regulations. The analysis reveals that advisors primarily support teachers into two aspects: educational planning with linguistic and cultural relevance, and strengthening teachers' teaching of mathematics, Spanish and sciences.

Keywords: Academic advice, intercultural education, primary education, cultural and linguistic diversity.

\section{Funciones y ámbitos de los asesores académicos para la diversidad lingüística y cultural}

\section{Introducción}

Dor décadas, la escuela indígena fue concebida como un vehículo para la castellanización y la integración de los grupos indígenas a la sociedad nacional en detrimento de su propia lengua y cultura (Barriga, 2010a; Corona, 2008; Hamel, 2008). Este propósito ha sido modificado y, en los últimos veinte años, las directrices de política para el sistema indígena han incluido en sus líneas de acción conceptos como equidad, justicia social, indigenismo de participación, respeto a la diversidad lingüística y cultural e inclusión (Secretaría de Educación Pública [SEP], 1988, 1995, 2001, 2007, 2011a).

La declaración constitucional de la aceptación de la diversidad étnica y lingüística en $1992^{1}$ posibilitó que, años después, el modelo pedagógico de atención edu-

1. La irrupción en la escena nacional del Ejercito Zapatista de Liberación Nacional otorgó visibilidad a los grupos étnicos y permitió incorporar demandas de los grupos originarios de nuestro país; una de ellas fue la promulgación en 2003 de la Ley General de los Derechos Lingüísticos de los Pueblos Indígenas. 
cativa fuera reformado, y desde 1999 opera el enfoque de educación intercultural bilingüe (SEP/Dirección General de Educación Indígena [DGEI], 1999). Aunque las escuelas indígenas utilizan los libros de texto nacionales, en la reciente reforma promulgada a partir de la publicación del Acuerdo 592 se introdujeron cambios para el tratamiento escolar de la pluriculturalidad. Uno de ellos fue la propuesta de los llamados Marcos curriculares para la educación indígena, y el otro, los Parámetros curriculares. El primero consiste en "una norma pedagógica, curricular y didáctica" (SEP, 2011a, p. 50) que, con base en diversos materiales de apoyo, permite a los docentes incorporar y trabajar aspectos históricos, legales, características y formas de atención de la educación indígena. El segundo establece las bases pedagógicas para la enseñanza de la lengua indígena e incluye una guía para la elaboración de los programas de estudio (SEP/DGEI, 2008). En términos generales, la propuesta incluye la asignatura de enseñanza de la lengua indígena y la enseñanza del español como segunda lengua, todo ello para cumplir con "el mandato constitucional de ofrecer una educación intercultural y bilingüe, y de avanzar hacia la construcción de una nación plural" (SEP, 2011a, p. 40).

La propuesta contenida en los Parámetros curriculares (SEP/DGEI, 2008) responde a la diversidad presente en México: 68 grupos etnolingüísticos (Instituto Nacional de Lenguas Indígenas [INALI], 2009) que representan un poco más del 10\% de la población del país (Comisión Nacional para el Desarrollo de los Pueblos Indígenas, 2006). El sistema educativo indígena tiene presencia en 24 estados de la república y, de acuerdo con la DGEI, en el ciclo escolar 2010-2011 se matricularon 840,683 alumnos. Los seis estados con el más alto porcentaje de atención en esta modalidad fueron: Chiapas (28.32\%), Oaxaca (17.12\%), Guerrero (11.17\%), Veracruz (9.35\%) y Puebla (8.71\%); los niños inscritos en las escuelas de Baja California sólo representan el o.98\% (SEP/DGEI, 2011).

La información disponible evidencia un crecimiento en la atención, pero también muestra diferencias significativas si se comparan los indicadores de rezago educativo del sistema nacional y los de la modalidad indígena. Por ejemplo, los índices de deserción y de reprobación de la educación primaria indígena duplican los porcentajes del nivel nacional; también la eficiencia terminal es menor (82.2\%) que la reportada por las escuelas no indígenas (91.7\%) (SEP/DGEI, 2011). Estas disparidades revelan una desigualdad en las oportunidades de acceso y permanencia entre los niños mexicanos.

Schmelkes (2010) señala que muchas veces se establece una correlación entre ausencia de calidad en el medio indígena con la falta de preparación de los docentes indígenas. Si bien es verdad que el ingreso al sistema de muchos docentes fue posi- 
ble por ser hablantes de una lengua indígena, independientemente de su formación académica, ${ }^{2}$ ése es uno de los muchos problemas que aquejan al sistema indígena y a la población indígena en su conjunto. De acuerdo con el INALI (2009), el analfabetismo de la población indígena $(25.4 \%)$ triplica el porcentaje aceptado a nivel nacional. Aunado a ello, se reconoce que el gasto en educación para la atención de estos grupos siempre ha sido inferior al recibido por otros sectores (Schmelkes, 2004); las condiciones de infraestructura de los planteles escolares no siempre han sido óptimas y en la organización escolar, aunque prevalecen escuelas que brindan los seis grados con igual número de profesores (54.81\%), aún se encuentran escuelas multigrado o atendidas por dos, tres, cuatro o cinco maestros (SEP/DGEI, 2011). Por otra parte, la profesionalización de los docentes indígenas es una tarea pendiente ya que, de acuerdo con la SEP/DGEI (2011), del total del profesorado en funciones (38,180 a nivel nacional), sólo $20.28 \%$ cuenta con estudios de educación superior (titulado).

En este contexto, una medida relevante para mejorar la calidad de este sistema lo constituye el Programa de asesores técnicos pedagógicos ${ }^{3}$ y para la atención a la diversidad social, lingüística y cultural (SEP, 2011b), programa que, de acuerdo con la documentación oficial, inició en 1998. Las autoridades educativas definen la asesoría como el "proceso de ayuda basado en la interacción profesional, en tres niveles, intervención, facilitación y colaboración, orientado a la resolución de problemas de una organización educativa” (SEP, 2010, p. 3). Debe señalarse que la asesoría pedagógica $\mathrm{y}$ académica se ofrece en todo el sistema educativo nacional; en el caso de la educación indígena, además del grupo de asesores asignados en las supervisiones escolares, cuenta con los asesores académicos que asumen como función primordial la atención a la diversidad lingüística, social y cultural. Así, la asesoría que se brinde a los docentes es fundamental y relevante no sólo para entender el sentido de las nuevas propuestas curriculares, sino para que la escuela se convierta en un espacio comunicativo privilegiado para revitalizar las lenguas originarias y atender el desarrollo lingüístico infantil en los años escolares (Barriga, 2010b).

2. Para el ingreso como docente en el sistema indígena se solicitó, en la década de los setenta, comprobar estudios de educación básica. A partir de 1990 era un requisito haber cursado estudios de bachillerato. Actualmente se exige la licenciatura terminada, además de participar en el proceso de selección que se hace a nivel nacional (Coordinación Estatal de Educación Indígena, 2012).

3. Las Reglas de Operación (SEP, 2011b) establecen la diferencia entre Asesores Técnicos Pedagógicos (ATP) y Asesores Académicos para la Diversidad (AAD). Los primeros eran quienes "asesoraban pedagógicamente a los docentes en educación indígena en el programa ATP”, y los segundos son "profesores comisionados para brindar apoyo académico a los docentes frente a grupo de las escuelas de educación indígena” (p.3). 
Las funciones que deben cumplir los asesores se encuentran enumeradas en las Reglas de operación ${ }^{4}$ que se emiten anualmente desde 2003 y que, por una parte, constituyen el marco regulador de los maestros que deseen ejercer esa actividad, y por otra, predeterminan su actuación al establecer funciones y actividades específicas. En dichas Reglas se estipulan los criterios de elegibilidad para quienes aspiran a incursionar como asesores (entre los más sobresalientes están el conocimiento de su contexto y ser hablantes de la lengua del grupo que atenderán, además de comprobar estudios de educación superior). Debido a que este programa otorga recursos económicos, las autoridades federales señalan de antemano el número de asesores autorizados para cada estado. Por su parte, las autoridades estatales pueden organizar tanto actividades de capacitación como compensar económicamente a los asesores. El apoyo económico que reciben los asesores los obliga a ofrecer y reportar ocho sesiones de asesoría mensuales. Finalmente, este programa, coordinado y dirigido por la DGEI, ha elaborado materiales que fundamentan, apoyan y guían las funciones y actividades que deben cumplir los asesores.

Uno de esos materiales es Bases generales para la función asesora. Perfil del ATP de Educación Indígena (2010) que define, a partir de establecer cuatro ámbitos de influencia (didáctico-áulico, organizativo-escolar, social-comunitario y asesoría y acompañamiento a las escuelas), las competencias que deben demostrar los asesores. Igualmente, sugiere los contenidos que deben dominar para ofrecer las asesorías diferenciando y cubriendo cada ámbito. En la última reforma a la educación básica (SEP, 2011a), uno de los principios pedagógicos que sustenta el plan de estudios, es el de la tutoría y la asesoría académica en la escuela. El documento rector define la asesoría como el "acompañamiento que se da a los docentes para la comprensión e implementación de las nuevas propuestas curriculares. Su reto es la resignificación de conceptos y prácticas" (p. 41). En el caso de la educación indígena, los asesores académicos, además, deben intervenir para solucionar la problemática que se presente en la escuela o su entorno.

\section{La asesoría académica}

La asesoría es una práctica que se realiza desde hace décadas en diferentes sistemas educativos del mundo. Debido a sus orígenes en otros campos de atención, más allá

4. El nombre del programa ha sido modificado en tres ocasiones. Inició como Reglas de operación e indicadores de gestión y evaluación del programa asesor técnico pedagógico (2003, 2004, 2006 y abril de 2007); en diciembre de 2009 cambió a Reglas de operación del programa Asesor Técnico Pedagógico, y en 2010 se le asignó el nombre de Reglas de operación del programa Asesor Técnico Pedagógico y para la atención educativa a la diversidad social, lingüística y cultural. En 2011 mantuvo esa denominación. 
de la discusión en torno a lo que define esta práctica en el ámbito educativo (Dean, 1997; Domingo, 2003; Rodríguez, 1996; Romero, 2006), se reconoce en la literatura sobre este tema (Dean, 1997) que quienes tienen asignada una labor de asesoramiento en el sistema escolar realizan una diversidad de prácticas, tienen sobrecarga de trabajo, llevan a cabo las actividades en circunstancias diversas, cubren un abanico amplio de responsabilidades y no hay una clara definición de sus tareas. También se afirma que debido a sus múltiples funciones, los asesores realizan su trabajo día a día "sin [...] llevar a cabo planificación alguna" (Dean, 1997, p. 18).

Rodríguez (1996) asevera que así como "la labor de asesoramiento ha surgido solapada en otras prácticas, las personas que la desempeñan también aprenden a ejercerla a partir de experiencias profesionales adquiridas" (p. 39). En ese sentido, se puede destacar que las prácticas de asesoría se configuran dependiendo del ámbito de influencia y, también, que en esta configuración influirá la formación de quien ejerce el cargo. De ahí que la literatura especializada le conceda importancia a la construcción del rol de asesor; Nieto (1993, citado en Romero, 2006) asevera que el asesoramiento puede ser concebido en tres vertientes: como una práctica institucionalizada, como una práctica individualizada y como una práctica personal. La primera estaría constreñida por componentes político-administrativos que son todos aquellos que norman tanto el sistema escolar como las condiciones de trabajo de los asesores; la segunda se relaciona con aquellos "componentes culturales e ideológicos de un contexto institucional que prioriza el asesoramiento individual y minimiza el trabajo colectivo" (p. 128), y la tercera se refiere a los componentes que marcan la biografía y trayectoria del asesor. Cada una de estas vertientes nos permite explicar las prácticas heterogéneas que pueden presentarse en la actividad de los asesores. En el contexto educativo mexicano, la práctica de los asesores puede ser considerada como emergente (Tapia, 2008).

Algunos autores que han indagado sobre esta figura en el sistema educativo (Calvo, 2007; López, Reyes \& Guerra, 2009) han identificado problemas que afectan su actividad y que son similares a los descritos en investigaciones en otros países: el personal designado es insuficiente; los asesores tienen escasa preparación académica para desarrollar esa función; además realizan diversas y numerosas actividades académicas y administrativas. También se reconoce que su permanencia en el puesto a veces es corta, por lo que los proyectos pedagógicos que se impulsan en los centros escolares se ponen en riesgo. Aunado a ello, los autores citados anotan que hay desconocimiento sobre las condiciones reales en las que desempeñan sus funciones, así como sobre la naturaleza de las prácticas y sus contextos de actuación (Calvo, 2007; Tapia, 2008). 
Un estudio de Meza, Vázquez y Cordero (2011) agrupó las funciones que los asesores tienen asignadas a partir del análisis de los documentos oficiales que delimitan esa tarea; concentró 36 posibles funciones relacionadas con cinco ámbitos de trabajo: formación continua, manejo de la dinámica escolar, diseño de programas de acuerdo con el contexto, atención de programas institucionales de apoyo y cuestiones vinculadas con la gestión escolar. Los resultados muestran, por una parte, una variedad de funciones encomendadas, y por otra, una cantidad considerable de documentación oficial que regula esta práctica. No hay duda que las funciones propuestas para que los asesores desempeñen su quehacer son centrales en las actividades cotidianas de las escuelas y los docentes (por ejemplo, apoyar a los docentes para la elaboración de proyectos didácticos de acuerdo con el contexto escolar) o bien, informar y capacitar al profesorado en los múltiples programas de apoyo vigentes en el sistema educativo (Programa Nacional de lectura, Escuela segura, Escuela y salud, Escuela siempre abierta, Ver bien para aprender mejor, entre otros). No obstante, la multiplicidad de funciones que encontraron las autoras desvirtúa el asesoramiento, en la medida que dista mucho de ser un proceso de acompañamiento por parte de los asesores a los docentes que les permita, de manera efectiva, resolver las situaciones que se les presentan en su ámbito áulico.

Sánchez, Cordero y Bocanegra (2009) indagaron la percepción que tienen los asesores adscritos a las supervisiones de zona escolar sobre el trabajo que desempeñan. En el estudio participaron 52 asesores de un municipio de Baja California y, a través de un cuestionario, se les interrogó sobre cómo se definían como asesores, cómo llegaron a ser asesores, qué acciones realizaban como asesores y qué tareas le resultaban más y menos satisfactorias. Los resultados muestran que los asesores se ven como facilitadores; $85 \%$ respondió que habían sido invitados por el supervisor de zona para fungir en ese puesto, y en la descripción de sus acciones mencionaron, entre otras, "asesorar y apoyar los programas de apoyo educativo, diseñar talleres, asesorar a maestros según problemáticas que se presentan en la docencia” (p. 7). Entre las tareas más satisfactorias anotaron: intervenir en el mejoramiento del proceso enseñanza aprendizaje $(31 \%)$, concientizar al maestro sobre su práctica docente $(24 \%)$, informar y asesorar sobre los programas de apoyo educativo (24\%), coordinar e impartir talleres para el intercambio de experiencias (14\%) y visitar escuelas (7\%). Entre las menos satisfactorias apuntaron las tareas administrativas.

Además, se cuenta con otras fuentes de información debido a que el programa de asesores técnico-pedagógicos ha sido evaluado regularmente por instituciones externas a la Dirección General de Educación Indígena. En 2007, la Universidad Pedagógica Nacional (UPN, 2007) reveló en su evaluación que el programa sí había cumplido 
una función necesaria para complementar la capacitación de los docentes en servicio, pero el programa no definía, ni clarificaba de forma suficiente, la manera en cómo las asesorías podrían ser un elemento nodal en la promoción del enfoque intercultural. La evaluación solicitada al Centro de Investigaciones y Estudios Superiores en Antropología Social coordinada por Nahmad (2009) subrayó que era necesaria una mayor profesionalización de los asesores, destinar mayor tiempo a las asesorías docentes, realizar evaluaciones cualitativas, reforzar el uso de las lenguas indígenas y proporcionar mayores insumos (bibliografía y materiales de apoyo) al personal docente. Por su parte, el estudio realizado por la Facultad Latinoamericana de Ciencias Sociales (FLACSO, 2008) describe con detalle el desarrollo del programa de asesorías del ciclo escolar 2007-2008; sin embargo, sólo recolectó información de 11 de las 24 entidades que operan el programa. Baja California no fue de los estados encuestados. Entre los resultados más sobresalientes está que no todo el profesorado recibió asesoría durante ese ciclo escolar; solo el $89 \%$ declaró haber asistido al menos a una asesoría. Del total de profesores que sí recibieron asesoría, el 62\% señaló sentirse satisfecho con el trato y el trabajo de los asesores, pero en el análisis de los mismos resultados por género, se observó discrepancia entre maestros y maestras: "mientras que 2 tercios de los docentes hombres se sienten satisfechos y muy satisfechos, el porcentaje entre las docentes es de poco más de la mitad de ellas (54.8\%). En todo caso, el 30\% de las docentes declararon [sic] sentirse regularmente satisfechas con el trato del ATP durante las asesorías recibidas" (FLACSO, 2008, p. 29). Este informe, entre muchos otros aspectos, consigna que un alto porcentaje de docentes $(87 \%)$ reveló haber mejorado su práctica docente como resultado de las asesorías. Otro dato relevante es que $98 \%$ de los encuestados manifestó conocer la existencia del programa de asesores, pero más de la mitad desconoce las reglas bajo las cuales opera.

Otros estudios realizados a propósito del programa de asesores técnicos pedagógicos son los de Juárez y Comboni (2007) y Comboni y Juárez (2009). Uno reporta la evaluación realizada al programa en el estado de Chiapas y el otro, similar en el tratamiento y la metodología empleada, muestra la actuación de los asesores en Oaxaca. Ambos estudios llegan a conclusiones similares: los asesores le otorgan mayor peso a la enseñanza del español que a la enseñanza de la lengua indígena, pero esto puede deberse a múltiples factores: desde el grado de bilingüismo del docente, su posición respecto a la utilidad de la lengua indígena, o el acceso a materiales en la variante de la región. Por otra parte, los investigadores también reportan que no se cumple con las asesorías establecidas en las Reglas de operación, pero en ambos estudios se señala que hay evidencia de que aquellos maestros que recibieron las asesorías introdujeron diferentes estrategias para abordar su trabajo, como impartir clases de biología en el 
campo o motivarlos para que expresaran abiertamente sus ideas a partir de las lecturas realizadas (Juárez \& Comboni, 2007).

\section{Contexto}

Baja California es un estado de la frontera norte de México; una de sus características es ser un polo de atracción para trabajadores migrantes. Aunque cuenta con población nativa, muchos de los niños que asisten a escuelas de la modalidad de educación intercultural bilingüe radican aquí debido a que sus padres encontraron alguna fuente de empleo (principalmente en labores agrícolas). En 2005, de acuerdo con cifras proporcionadas por el Instituto Nacional de Estadística y Geografía (INEGI, 2010), se identificaron hablantes de 13 lenguas en el estado. Este instituto reportó que $12 \%$ de 5,131 niños indígenas de cinco a 14 años eran monolingües, y que la tasa de asistencia escolar de los niños de ese rango de edad era del 76.5\%. La información muestra que conforme aumenta la edad, se incrementa su asistencia a la escuela (50\% a los cinco años, $89.4 \%$ a los nueve años), pero al paso de los años vuelve a disminuir (6o\% de asistencia a los 14). Igualmente, el reporte consigna que sólo $76.7 \%$ de los niños de ocho años que habla alguna lengua indígena sabía leer y escribir (español), porcentaje que aumenta al $93 \%$ a los 12 años.

Desde mi perspectiva, estas cifras ponen en evidencia dos problemas que los profesores enfrentan en esta modalidad en el estado de Baja California: diversidad lingüística y monolingüismo en una lengua que no es la suya. Desde los años ochenta, cuando inició la atención a la demanda de población indígena en el estado, ha ido en aumento el número de alumnos, escuelas y docentes de primaria. De acuerdo con la SEP/DGEI (2011), en el ciclo escolar 2009-2010 funcionaban 60 centros escolares atendidos por 441 profesores y 9,862 alumnos inscritos. Según datos de la Coordinación Estatal de Educación Indígena de Baja California (2012), los maestros en funciones pertenecen a 14 etnias diferentes, aunque la mayoría (76.4\%) es de origen mixteco; el $73 \%$ de los maestros cuenta con licenciatura, normal básica o maestría, pero $27 \%$ todavía requiere concluir estudios superiores. En el sistema estatal hay una proporción mayor de maestros (65.31\%) que de maestras (34.69\%). El número de asesores académicos ha ido en aumento. En 2007 fueron autorizados cinco asesores y en 2011 estaban trabajando 11 (nueve para atender primaria y dos para preescolar). Cada año se emite una convocatoria para concursar por ese tipo de nombramiento, por ello su permanencia como asesores sólo es por ese periodo. Si aspiran a permanecer en ese puesto, deben cumplir con los requerimientos estipulados y esperar a ser designados por otro periodo. 


\section{Metodología}

El objetivo que tuvo este estudio fue documentar las funciones y actividades que los Asesores Académicos para la Diversidad (AAD) realizan en los centros escolares. Para conocerlas, se recopilaron los informes semestrales presentados por los asesores a la Coordinación Estatal de Educación Indígena. ${ }^{5}$ En total se tuvo acceso a 490 informes de nueve AAD de primaria en 2011. De los nueve asesores que tenían ese nombramiento, dos trabajaron todo el año y siete sólo por un semestre. Debe señalarse que, en su conjunto, los informes son breves: en pocas líneas los asesores describen la problemática, la intervención, los resultados y las sugerencias. En teoría, un asesor debe entregar 40 informes por semestre; no obstante, el número de informes reportado es mayor al establecido, ya que muchos asesores elaboraron más de los solicitados.

Los informes fueron capturados en una base de datos; para el análisis, se retomó la propuesta de Tójar (2006) de análisis de contenido deductivo. Las funciones enumeradas en las Reglas de operación sirvieron como categorías generales, y a partir de la lectura repetida de la problemática enunciada se agruparon y clasificaron. El proceso de análisis también permitió tomar en cuenta categorías emergentes (inductivas). Otro elemento considerado fueron las particularidades de los informes, como diferencias y similitudes entre el total de informes, extensión, número de profesores beneficiados, alumnos y formas de intervención predominantes.

\section{Resultados}

Todos los asesores académicos de primaria designados en 2011 demostraron tener estudios de licenciatura y ser hablantes de su lengua (mixteco). Dos docentes han sido asesores desde el inicio del programa (1998) y el resto había ingresado en las dos últimas convocatorias. En cuanto a su antigüedad en el sistema educativo, se encontró que cinco tienen entre uno y 10 años, y el resto entre 11 y 20 años.

5. Las Reglas de operación (SEP, 2011b) indican que los AAD están comprometidos a cumplir ocho asesorías por mes y deben elaborar un informe de cada una. Por su parte, las autoridades proporcionan un formato para la captura de dicha información; en éste describen la problemática detectada, cómo procedieron para resolverla (intervención), los resultados obtenidos y sugerencias para futuras asesorías. También deben mostrar evidencia de que fueron efectuadas las asesorías; esto se constata a través de las firmas de los profesores que asistieron a la sesión. El informe debe contener también el visto bueno del director y supervisor de la zona escolar. Finalmente, los asesores deben remitir los informes al Coordinador de los Asesores Académicos (CAAD) para su revisión y seguimiento de las prácticas de asesorías. 
Los informes de los asesores son elaborados a partir de una plantilla proporcionada por la DGEI, en donde anotan qué tipo de problemática atendieron, la intervención que llevaron a cabo, qué resultados obtuvieron y recomendaciones generales. Igualmente, deben incluir información sobre el número de profesores y alumnos beneficiados. La caracterización de los informes ofrece pistas sobre la forma en que los AAD abordan las asesorías académicas, la problemática en las escuelas que atienden, pero, sobre todo, en el tipo de estrategias que utilizan para solucionarlas. Este aspecto cobró relevancia al contabilizar algunas formas de intervención (Tabla 1).

\section{Tabla 1. Formas de intervención utilizadas por los ADD}

\begin{tabular}{lc}
\hline \multicolumn{1}{c}{ Formas de intervención } & \% \\
\hline Taller & 25.84 \\
\hline Asesoría al docente & 31.13 \\
\hline Revisión y análisis & 22.12 \\
\hline Planeación & 9.76 \\
\hline Observación y seguimiento & 9.45 \\
\hline Diagnóstico & 5.57 \\
\hline Capacitación docente & 2.02 \\
\hline
\end{tabular}

Fuente: Elaboración propia a partir de los datos.

Los informes son de muy diferente extensión; en términos generales, todos son breves, pero algunos de ellos describen en pocas líneas la problemática detectada y con una palabra enuncian la forma de intervención. Ejemplo: Taller (AAD1.A.En). ${ }^{6}$ Otros informes son más detallados, no sólo en la descripción de la problemática sino en cómo la abordaron:

Se detectó que en la escuela, la vinculación y secuencia didáctica es prioridad en el diseño y aplicación de la planeación didáctica atendiendo la articulación de los contenidos que se aplica en el aula. Para atender esta problemática se implementó el taller de: 2 da jornada del tema curricular y la relevancia social en consejo técnico consul-

6. Los ejemplos que se transcriben corresponden a los informes elaborados por los asesores en 2011. Para el control de las transcripciones se les asignó un número a los maestros, una letra por el periodo ( $\mathrm{A}=$ Anual y $\mathrm{S}=$ Semestral) y la abreviación del mes en el que reportó la asesoría. 
tativo, como un espacio para compartir los conocimientos y experiencias docentes.? (AAD5.S.Dic)

En los informes se incluyen datos como número de escuelas atendidas, profesores y alumnos. Otra característica sobresaliente de los informes es la especificación de los beneficiados; por ejemplo: si la asesoría fue proporcionada al directivo ("Elaboración de instrumentos de evaluación para alumnos de quinto grado a nivel zonal"), el asesor anotó: una escuela, un directivo, 208 alumnos (AAD2.A.Feb), aunque debe hacerse notar que, en este caso particular, el reporte de asesoría era específico para un grado escolar pero incluía a los niños de zona escolar. En otros casos, la asesoría sí involucra a un número considerable de maestros, como en el siguiente ejemplo: "Conocimiento, reconocimiento, valoración y aprecio de la diversidad en el aula. Análisis de los marcos jurídico-normativos de la diversidad y la educación intercultural bilingüe, en los ámbitos nacional e internacional"; beneficiados: una zona escolar; 21 docentes; 500 alumnos (AAD10.A.May).

En relación con el diagnóstico de la problemática, debe hacerse notar que los informes revisados no aclaran, en muchas ocasiones, a qué obedeció la asesoría, como se muestra a continuación. Un asesor anotó: "Marco teórico de los parámetros curriculares" (AAD1.A.En). Este enunciado no permite reconocer si el asesor ofreció capacitación sobre los nuevos materiales o si hay poca comprensión de los docentes sobre ellos. En contraste, otro escribió: "Análisis e interpretación de los resultados de la evaluación aplicada a los alumnos de primer ciclo” (AAD2.A.Mar). Al señalar los resultados, el asesor escribió: "Se logró que cada docente reconociera el avance de sus alumnos en cada una de las asignaturas, identificando contenidos de mayor frecuencia de error". Esta anotación permite entender que el diagnóstico realizado por el asesor se relaciona con la inexperiencia de los docentes en la interpretación de los resultados de logro educativo de sus alumnos. Por otra parte, se desconoce el tiempo que le dedican a una asesoría; ese dato, importante a mi juicio, no está reportado en los informes. Por otra parte, se destaca un predominio de asesorías colectivas; del total, sólo el $35.91 \%$ fueron individuales.

7. Algunos ejemplos utilizados muestran problemas de sintaxis, pero se decidió respetar la redacción de los maestros. 


\section{Funciones y actividades}

En las Reglas de operación vigentes para 2011, se formularon 10 funciones ${ }^{8}$ que debían desempeñar los AAD (Ver Tabla 2); el análisis mostró que, aunque algunas funciones predominan, todos los asesores actúan de acuerdo con lo establecido institucionalmente. Además de éstas, los asesores desempeñan otras dos: una relacionada con la revisión de los fundamentos del enfoque intercultural bilingüe, y otra vinculada a la difusión de los programas de apoyo que operan en el sistema educativo.

\section{Tabla 2. Funciones establecidas en las reglas de operación 2011}

\begin{tabular}{|c|c|c|c|}
\hline & Funciones & Número & $\%$ \\
\hline 1 & $\begin{array}{l}\text { La Reforma Integral de Educación Básica y la articulación de la } \\
\text { Educación Básica Indígena. }\end{array}$ & 18 & 3.67 \\
\hline 2 & La planeación didáctica con pertinencia lingüística y cultural. & 113 & 23.06 \\
\hline 3 & $\begin{array}{l}\text { La incorporación de elementos que favorezcan el currículo, a partir } \\
\text { del contexto cultural y lingüístico local. }\end{array}$ & 10 & 2.04 \\
\hline 4 & $\begin{array}{l}\text { El uso social de la lengua indígena como medio de comunicación y } \\
\text { como objeto de estudio. }\end{array}$ & 1 & .2 \\
\hline 5 & $\begin{array}{l}\text { El uso y aprovechamiento de los materiales educativos (tanto en } \\
\text { lengua indígena como en español). }\end{array}$ & 22 & 4.48 \\
\hline 6 & $\begin{array}{l}\text { El fortalecimiento de la didáctica de enseñanza de las matemáticas, el } \\
\text { español y las ciencias. }\end{array}$ & 144 & 29.38 \\
\hline 7 & $\begin{array}{l}\text { Identificar y proponer estrategias innovadoras de evaluación de los } \\
\text { aprendizajes. }\end{array}$ & 53 & 10.81 \\
\hline 8 & Promover la profesionalización de las y los docentes. & 18 & 3.46 \\
\hline 9 & Orientar la elaboración y gestión del proyecto escolar. & 50 & 10.2 \\
\hline \multirow[t]{2}{*}{10} & El fortalecimiento del trabajo colegiado y del aprendizaje cooperativo. & 4 & .81 \\
\hline & Funciones adicionales & & \\
\hline 11 & Revisión de los fundamentos del enfoque intercultural bilingüe. & 18 & 3.46 \\
\hline \multirow[t]{2}{*}{12} & Información sobre programas de apoyo. & 39 & 7.95 \\
\hline & TOTAL & 490 & 100 \\
\hline
\end{tabular}

8. Las funciones han sido modificadas en cada emisión de las reglas de operación. En 2003 se enunciaron 11; en 2004 y 2006, 13; en 2007, 14; en 2008, 10; en 2009, 11; y en 2010 y 2011, 10. Tres de ellas, las relacionadas con la lengua indígena, han permanecido. 
Los asesores dedican parte de su tiempo a apoyar a los profesores en cuestiones relacionadas con la didáctica de la enseñanza tanto de las matemáticas como del español (29.38\%). Igualmente, realizan actividades para que los docentes elaboren planeaciones didácticas con pertinencia lingüística y cultural (23.06\%). Debe anotarse que los informes de los asesores reportan que dicha planeación está vinculada con las orientaciones contenidas en el documento de Lengua indígena. Parámetros curriculares, pero se ubicó que asesoran a los docentes sobre los fundamentos del enfoque intercultural (3.46\%).

Otra función destacada es la Orientación en la elaboración y gestión del proyecto escolar. En este caso, las asesorías documentadas se relacionan con el proyecto escolar que cada colectivo escolar debe presentar anualmente, pero debe destacarse que los AAD dedican una parte de su tiempo a difundir los programas de apoyo vigentes o emergentes, por ejemplo, Enciclomedia, escuela segura, programa de salud, programa de lectura, etc., o bien anotan que trataron problemas que aquejan a la comunidad (adicciones).

Por otra parte, hay funciones que los asesores no realizan asiduamente; tal es el caso de la promoción del trabajo colegiado (.81\%), el uso social de la lengua indígena como medio de comunicación y como objeto de estudio (.2\%), y el uso y aprovechamiento de materiales educativos (4.28\%). En el caso de la función 7 (10.81\%), los asesores analizan los procesos de evaluación, junto con los maestros, a partir de los resultados obtenidos por los alumnos en las pruebas de logro educativo que periódicamente se llevan a cabo. Al analizar la distribución de las asesorías por función y por asesor, es notorio que muchos de ellos no desempeñan las funciones enumeradas en las Reglas de operación (Tabla 3).

Es importante destacar algunos casos; de todos, el trabajo del AADı cubrió, en diferente medida, todas las funciones (es uno de los docentes con mayor antigüedad en el programa). El resto concentró su actividad en algunas de ellas y algunos asesores ( $A_{4} \mathrm{y}_{4} \mathrm{AAD}_{7}$ ), de manera significativa, se abocaron a realizar contadas funciones. Las asesorías del $\mathrm{AAD}_{4}$ estuvieron relacionadas con la función 2 (todos sus informes describen actividades de planeación a partir de la propuesta de Parámetros curriculares, tres de ellas las dedicó a ofrecer información sobre la fundamentación del enfoque intercultural bilingüe).

La enseñanza de la lengua materna debe ser la función de mayor peso en la escuela indígena. Hay un énfasis institucional para que los asesores realicen actividades vinculadas a ella, y si bien todos realizan actividades orientadas a que el docente domine la propuesta de elaboración de programas de estudio de enseñanza de la lengua indígena a partir de lo que marca el documento de Parámetros curriculares, también es claro que son contadas las alusiones a la lengua indígena como medio de comunicación o a la lengua como objeto de estudio. Los asesores han recibido cursos sobre 
Parámetros curriculares por parte de la DGEI, y ellos deben capacitar a los docentes, pero puede inferirse que si el asesor no tiene una comprensión de la fundamentación para elaborar los programas de estudio de la lengua indígena, es probable que no la aborde en sus sesiones.

Tabla 3. Funciones realizadas por el asesor académico

\begin{tabular}{ccccccccccccccc}
\hline & & & \multicolumn{10}{c}{ Funciones } \\
\hline AAD & $\begin{array}{c}\text { No. de } \\
\text { informes }^{*}\end{array}$ & $\begin{array}{c}\text { Asesorías } \\
\text { individuales }\end{array}$ & $\mathbf{1}$ & $\mathbf{2}$ & $\mathbf{3}$ & $\mathbf{4}$ & $\mathbf{5}$ & $\mathbf{6}$ & $\mathbf{7}$ & $\mathbf{8}$ & $\mathbf{9}$ & $\mathbf{1 0}$ & $\mathbf{1 1}$ & $\mathbf{1 2}$ \\
\hline 1 & 79 & 47 & 5 & 5 & 6 & 1 & 18 & 22 & 13 & 6 & 2 & 2 & 0 & 0 \\
\hline 2 & 79 & 32 & 5 & 4 & 1 & 0 & 1 & 15 & 20 & 2 & 15 & 2 & 0 & 10 \\
\hline 3 & 32 & 16 & 2 & 8 & 1 & 0 & 0 & 12 & 1 & 0 & 0 & 0 & 0 & 8 \\
\hline 4 & 32 & 18 & 0 & 29 & 0 & 0 & 0 & 0 & 0 & 0 & 0 & 0 & 3 & 0 \\
\hline 5 & 32 & 16 & 0 & 3 & 0 & 0 & 0 & 13 & 7 & 1 & 5 & 0 & 3 & 0 \\
\hline 6 & 47 & 1 & 0 & 9 & 0 & 0 & 0 & 15 & 0 & 0 & 20 & 0 & 3 & 0 \\
\hline 7 & 92 & 19 & 0 & 36 & 0 & 0 & 0 & 33 & 10 & 3 & 7 & 0 & 3 & 0 \\
\hline 8 & 50 & 1 & 1 & 14 & 0 & 0 & 0 & 14 & 0 & 3 & 1 & 0 & 0 & 17 \\
\hline 9 & 47 & 25 & 4 & 4 & 3 & 0 & 2 & 19 & 2 & 3 & 0 & 0 & 6 & 4 \\
\hline Total & 490 & 175 & 18 & 113 & 10 & 1 & 22 & 144 & 53 & 18 & 50 & 4 & 18 & 39 \\
\hline
\end{tabular}

Fuente: Elaboración propia a partir de la información recabada.

* El número de informes varía. Del total de asesores, dos estuvieron en el puesto todo el año, el resto sólo trabajó un semestre. Sobresale el AA7 que remitió 92 informes; su obligación, de acuerdo con la normativa del programa, era consignar 40 reportes.

Por otra parte, se debe tomar en cuenta que la labor del asesor, además de estar predeterminada por las Reglas de operación, responde al contexto donde se desempeña. En ese sentido, su actuación depende del diagnóstico sobre las escuelas o las necesidades de los profesores. Sobre esta base pueden tomar decisiones en torno a qué aspectos abordar de manera prioritaria, pero centrarse en determinadas funciones corre el riesgo de relegar aspectos que necesitan ser fortalecidos en las escuelas, como el impulso de los colectivos escolares, o bien, la articulación de la última reforma de educación básica con los postulados de la educación intercultural. Por ejemplo, en la reforma se postula como principio pedagógico Favorecer la inclusión para atender la diversidad, pero ya no se pone énfasis en el concepto de interculturalidad (SEP, 2011a). 


\section{Ámbitos de competencia de la asesoría académica}

Uno de los materiales de apoyo para la asesoría académica en la escuela indígena lo constituye el documento elaborado por la SEP/DGEI Bases generales para la función asesora. Perfil delATP deEducación Indígena (2010); en éste se enuncian las competencias que debe poseer el asesor, las cuales están agrupadas en cuatro ámbitos: didáctico áulico, organizativo-escolar, social-comunitario y asesoría, y acompañamiento a las escuelas de educación indígena. Además, a cada competencia le corresponden varias subcompetencias, contenidos y materiales que debe dominar. Algunos ejemplos se muestran en la Tabla 4.

Tabla 4. Competencias, contenidos y materiales para los AAD

\begin{tabular}{lll}
\hline \multicolumn{1}{c}{ Ámbito: Didáctico áulico } & & \multicolumn{1}{c}{ Ejemplos } \\
\hline \multicolumn{1}{c}{ Competencia } & Subcompetencia & \multicolumn{1}{c}{ Contenidos y materiales } \\
\hline Indaga los resultados de logro & 1. & Evaluaciones externas del aprendizaje \\
académico de las escuelas que & 2. & internacionales (PISA) y nacionales (ENLACE). \\
asesora y observa la práctica & 3. & Uso de la información de evaluaciones externas. \\
de los maestros y maestras & 4. & Uso y manejo de Internet. \\
para promover procesos de & & Técnicas cualitativas de recolección de \\
revisión crítica de la práctica. & & información (registros de observación). \\
\hline
\end{tabular}

Fuente: SEP/DGEI (2010). Bases generales para la función asesora. Perfil del ATP de Educación Indígena (pp.19-2o).

A partir de la categorización de la problemática enunciada por los AAD se identificaron los ámbitos predominantes en la función asesora; se encontró que están relacionados con los tres primeros. En los informes no se reportan actividades que correspondan al ámbito social comunitario (Tabla 5).

\section{Tabla 5. Ámbitos de competencia presentes en los informes de seguimiento}

\begin{tabular}{lcc}
\hline \multicolumn{1}{c}{ Ámbito } & Número (490) & \% \\
\hline Didáctico áulico & 379 & 77.34 \\
\hline Organizativo-escolar & 18 & 3.67 \\
\hline $\begin{array}{l}\text { Asesoría y acompañamiento a las } \\
\text { escuelas de educación indígena }\end{array}$ & 93 & 18.97 \\
\hline
\end{tabular}


En el ámbito didáctico áulico se les solicita la revisión de los resultados de logro académico de las escuelas y la promoción de procesos de revisión crítica de la práctica. En ese sentido, predominan sesiones donde se señala que:

Se revisó en colectivo los resultados bimestrales comprendidos de septiembre a diciembre 2010 [...] Se analizó con el colectivo los resultados de enlace 2010, para identificar el nivel de desempeño de logro académico [...] Se definieron estrategias de intervención para mejorar los resultados de aprovechamiento escolar y las acciones que habrán de analizarse en colectivo en tiempos determinados. (AAD2.A.Feb)

O bien "Dentro de la zona escolar hace falta redefinir el enfoque del proyecto escolar en la que se centre la atención en el logro educativo y partir de ella, mejorar los índices de desempeño de los alumnos" (AAD7.S.Sep).

Igualmente, se les solicita que apoyen a los docentes bilingües en la elaboración de una planeación didáctica acorde al enfoque intercultural. Los asesores señalan: "Dentro de la zona escolar hace falta definir parámetros para abordar la asignatura: Lengua indígena en el trabajo por proyectos de 1 y 2 grados" (AAD6.S.Sep).

Se detectó que las decisiones que toma el docente al diseñar las secuencias didácticas son de vital importancia, por lo que es preciso familiarizarse con el plan y programa de estudios 2011 de la educación primaria, para que la intervención docente y sus aplicaciones en el aula sea la más acertada de acuerdo a las necesidades educativas de los alumnos. (AAD5.S.Oct)

O bien, reportan sugerencias a los docentes sobre el desarrollo de estrategias didácticas:

Se llevó a cabo al [sic] acompañamiento pedagógico a la docente en la que se le sugirió retomar la vinculación de los aprendizajes esperados de parámetros curriculares con la RIEB en la planeación didáctica dándole algunas estrategias a utilizar en su práctica. (AADı.S.Feb)

Respecto al ámbito organizativo escolar, una competencia marca que se debe orientar a maestras y maestros bilingües, así como a directores, en la elaboración de una planeación escolar acorde con las necesidades de la escuela indígena. Muchos de los docentes hacen alusión a ello, aunque no clarifican del todo lo que realizan, como en el siguiente ejemplo: "Desconocimiento sobre el propósito general del parámetro curricular y la planeación de algunas actividades enfocadas a la lengua indígena" (AAD8.S.Mar). Respecto al trabajo colegiado entre directores, maestras y maestros, son pocos los casos a los que se alude, pero están relacionados con el "Análisis de las competencias y los estándares del desempeño docente” (AAD9.S.Jun).

Las autoridades educativas le conceden suma importancia al ámbito de asesoría y acompañamiento a las escuelas de educación indígena, y por ello se requiere que los 
$\mathrm{AAD}$ apliquen los principios de una asesoría orientada a la mejora educativa de las escuelas. Uno de los asesores informó que:

Se participó en el análisis de las funciones que brinda el asesor técnico en la reforma integral de educación básica, en la cual se analizó muy puntualmente cómo debe de acompañar al asesor ante sus asesorados teniendo en cuenta que debe de ser innovador, respetuoso, crítico y llevar una buena guía al docente para seguir mejorando su labor. (AAD1o.S.Feb)

En la identificación de la problemática presente en la escuela, los asesores hacen alusión a cuestiones sobre el entorno (adicciones, por ejemplo), o bien, cuestiones de índole administrativa (trámite de actas de nacimiento, alumnos extra edad o alumnos con necesidades educativas especiales). También destacan informes donde la asesoría se centró en la difusión de los programas de apoyo.

\section{Conclusiones}

El análisis de los documentos que norman las funciones y actividades de los asesores académicos en la modalidad de educación indígena evidencia varios problemas asociados al trabajo de estos. En primer lugar, debido a que los docentes concursan anualmente por el puesto, existe una rotación de personal que puede ocasionar interrupción en los proyectos pedagógicos y curriculares impulsados dentro de los colectivos escolares. En segundo lugar, el número de $\mathrm{AAD}$ aún es limitado para el trabajo que implica una asesoría eficaz en las escuelas; esto es, para que cubra todos los aspectos que deben ser tomados en cuenta y así cumplir con las nuevas orientaciones sobre la enseñanza de la lengua materna y la planeación didáctica propuesta por la reforma educativa instrumentada en 2011; tareas que ocuparían un primerísimo lugar en el asesoramiento docente, de acuerdo con los postulados de atención a la diversidad. En tercer lugar, el programa está regulado por un formato: las Reglas de operación, que definen la actuación del asesor al enumerar funciones específicas y actividades concretas, pero este formato, modificado anualmente, permite a la administración central consignar otras funciones que pueden convertirse, a la larga, en distractores que impidan el cumplimiento de las metas iniciales. Considero que sería más consecuente mantener un número limitado de funciones y que éstas, una vez satisfechas las demandas por parte del profesorado, podrían ampliarse o modificarse. También es importante mencionar que las Reglas de operación no son el único referente que tienen los asesores para realizar su labor; existen otros documentos difundidos por la DGEI que amplían los ámbitos de intervención, y acotan contenidos y materiales a partir del establecimiento de competencias y subcompetencias. 
Las actividades derivadas de las funciones que deben cumplir son vastas y heterogéneas; algunas de ellas pueden referirse como distintivas del asesoramiento en la modalidad indígena (todas las que se relacionan con la revitalización de las lenguas indígenas), y otras son más de tipo administrativo (como la elaboración misma del informe para comprobar que realizan las asesorías comprometidas o información sobre una serie de programas de apoyo, que se demanda institucionalmente sean difundidos entre los maestros). Las características predominantes de los informes son la brevedad, una descripción similar de contadas problemáticas y también un número de formas de intervención y de trabajo con los docentes. El informe es además un producto de control y de verificación por parte de las autoridades en la medida que se otorgan recursos económicos.

En sus reportes, los AAD se limitan a la enunciación de una problemática, y en ocasiones no concuerda ésta con la forma en como fue abordada, ni con los resultados, sugerencias o población beneficiada. Éste es un aspecto que de manera particular la Coordinación Estatal de Educación Indígena está atendiendo, pero una posible explicación se relaciona, por una parte, con el entendimiento de la función, y por otra, con la idea de que el informe es un trámite y no refleja del todo las múltiples tareas que realizan.

En algunos de los informes no hay evidencia clara de que dichas asesorías brindaron el apoyo requerido por los docentes; esto es, no se consigna si la asesoría fue a solicitud del director o del profesorado. Los estudios que han indagado la figura del asesor (Juárez \& Comboni, 2007; Comboni \& Juárez, 2009) y las evaluaciones externas permiten señalar que aún es necesario indagar con mayor profundidad la experiencia de los docentes en el asesoramiento recibido.

No obstante, a partir de las descripciones elaboradas por el asesor, es posible advertir el tipo de función que cumple prioritariamente, y conocer las múltiples actividades en contextos específicos, así como las necesidades que presentan las escuelas y el profesorado. En este sentido, los informes de los asesores académicos revelan información valiosa que puede ayudar a impulsar procesos de formación continua más focalizados.

Una de las observaciones centrales de la evaluación de la UPN de 2007 hacía notar que no se definía con claridad en el programa de qué manera podía impulsarse el enfoque que distingue a esta modalidad: el intercultural bilingüe. Si bien es cierto que las Reglas de operación de los dos últimos años han colocado el énfasis en la competencia lingüística del profesor y en la enseñanza de la lengua, también lo es que, por décadas, la política en el ámbito educativo pretendió la homogeneización de la población mediante la enseñanza del español como lengua nacional. No obstante, en la re- 
visión de las funciones sí aparecen aquellas relacionadas con la planeación didáctica a partir del documento de Lengua Indígena. Parámetros curriculares, aunque muchas de las asesorías, según reportan los asesores, se realizaron para que los maestros tengan un conocimiento detallado de las actividades que se proponen.

El análisis reveló que predomina la atención por parte de los asesores en aspectos relacionados con la planeación didáctica de la lengua indígena, y apoyo para cuestiones de la didáctica de la enseñanza del español y las matemáticas, pero emergieron funciones no contempladas institucionalmente, como la que implica la difusión de los programas de apoyo. En el sistema educativo existe un número considerable de programas -relacionados con las tecnologías, la promoción de la lectura, la seguridad de la escuela, la calidad o la salud- que tienen objetivos variados y diversos y que deben tener un seguimiento para su adecuación en las escuelas. No se puede asegurar que todos los asesores sean expertos en cada uno de ellos; sin embargo, la difusión de estos programas es parte de la labor que llevan a cabo. Por otra parte, aunque no es muy alto el porcentaje de asesorías individuales que organizan, destinan parte de las ocho asesorías mensuales a trabajar con un solo maestro, y se advierte en los informes que los temas abordados de manera individual se repiten con varios de ellos.

Sin duda, los programas de asesoría al profesorado son necesarios, sobre todo en contextos en donde está presente la marginalidad económica y social. La propuesta de asesoramiento para el mejoramiento educativo a través de los $\mathrm{AAD}$ es viable, pero es pertinente tomar en cuenta los contextos, la ubicación geográfica, la organización, el número y las prioridades de las escuelas. En Baja California hay presencia en las aulas de niños mixtecos, mixes, zapotecos, nahuas, triquis, entre otros. Hay zonas escolares que supervisan escuelas en un radio de 30 kilómetros, y el acceso a estos planteles se dificulta, muchas veces, por las condiciones climatológicas.

Finalmente, los informes sólo constituyen una parte del conjunto de actividades que realiza el asesor; no necesariamente reflejan el trabajo colegiado que han realizado con el profesorado, cómo han ido configurando su práctica, ni las múltiples tareas que realizan para organizar y planificar su asesoría. Es necesario llevar a cabo estudios in situ para reconocer qué implica la asesoría en la modalidad indígena. Es necesario conocer qué opinión tienen los docentes sobre el asesoramiento en temas que son cruciales para el impulso de un modelo intercultural que no sólo respete la diversidad lingüística presente en el país, sino que impulse la enseñanza de la lengua indígena. 
María Guadalupe Tinajero Villavicencio

\section{Lista de Referencias}

Barriga, R. (2010a). Una hidra de siete cabezas y más: la enseñanza del español en el siglo XX mexicano. En R. Barriga \& P. Martín (Dirs.), Historia Sociolingüística de México (Vol. 2, pp. 1095-1194). México: El Colegio de México.

Barriga, R. (2010b). El desarrollo de la lengua en los años escolares en contextos indoamericanos bilingües. En F. Palemón (Coord.), Por y para el florecimiento de la lengua náhuatl en la región de la Montaña Baja de Guerrero (pp. 17-21). México: Secretaría de Asuntos Indígenas de Guerrero-Consejo Nacional para la Cultura y las Artes.

Calvo, B. (noviembre, 2007). Los asesores técnico-pedagógicos y el fortalecimiento de las escuelas de educación básica. Memorias Electrónicas del IX Congreso Nacional de Investigación Educativa, Yucatán, México: Consejo Mexicano de Investigación Educativa.

Comboni, S., \& Juárez, J. M. (septiembre-diciembre, 2009). Los asesores técnico-pedagógicos y la educación intercultural en Oaxaca. Preliminares de una evaluación. Decisio, 24. Recuperado de http://tumbi.crefal.edu.mx/decisio/index. php?option $=$ com content $\&$ view $=$ article $\&$ id $=157 \&$ Itemid $=105$

Comisión Nacional para el Desarrollo de los Pueblos Indígenas. (2006). Programa de las Naciones Unidas para el Desarrollo. Informe sobre Desarrollo Humano de los Pueblos Indígenas de México. México: CDI-PNUD. Recuperado de http:// www.cdi.gob.mx/idh/informe_desarrollo_humano_pueblos indigenas mexico 2006.pdf

Coordinación Estatal de Educación Indígena (2012). Plan institucional 2012. Manuscrito inédito.

Corona, S. (2008). Políticas educativas y libros de la SEP indígena. Fronteras Educativas. Comunidad Virtual de la Educación. Recuperado de http://www.sinectica. iteso. $\mathrm{mx} /$ ?seccion $=$ articulo\&lang $=$ es $\& \mathrm{id}=443$ _politicas_educativas_y_libros de la sep_para indigenas

Dean, J. (1997). Supervisión y asesoramiento. Manual para inspectores, asesores y profesorado asesor. Madrid: La Muralla.

Domingo, J. (2003). El asesoramiento a centros educativos, una cuestión de saber, poder e identidad. Profesorado: Revista de Currículum y Formación del Profesorado, vol. 7 (1-2), pp. 103-112. Recuperado de http://www.ugr.es/ recfpro/ rev71COL1.pdf

Facultad Latinoamericana de Ciencias Sociales. (2008). Percepción de beneficiarios. México: Autor. Recuperado de http://basica.sep.gob.mx/dgei/pdf/evalua- 
Funciones y ámbitos de los asesores académicos

para la diversidad lingüística y cultural

María Guadalupe Tinajero Villavicencio

cion/Resultadosevaexo8.pdf

Hamel, R. E. (2008). Bilingual Education for Indigenous Communities in México. En J. Cummins \& N. H. Hornberger (Eds.), Encyclomedia of Language an Education (2a. ed., Vol. 5, pp. 311-322). New York: Springer.

Instituto Nacional de Estadística y Geografía. (2010). Estadísticas a propósito del día del niño. México: Autor Recuperado de http://www.inegi.org.mx/inegi/contenidos/espanol/prensa/Contenidos/estadisticas/2013/ni\%C3\%B10o.pdf

Instituto Nacional de Lenguas Indígenas. (2009). Programa de revitalización, fortalecimiento y desarrollo de las lenguas indígenas nacionales, 2008-2012. México: SEP-INALI.

Juárez, J. M., \& Comboni, S. (2007). La educación en el medio indígena modelo de neodominación o promesa de liberación. El Cotidiano, 22 146, 61-72. Recuperado de http://www.elcotidianoenlinea.com.mx/numeros.asp?edi=146

López, Y., Reyes, N., \& Guerra, J. (septiembre, 2009). Diagnóstico de la asesoría técnico-pedagógica y formación de recursos humanos de alto nivel en educación básica hacia el diseño y aplicación de un modelo de asesoría en el estado de San Luis Potosí. Memorias electrónicas del X Congreso Nacional de Investigación Educativa, Veracruz, México. Recuperado de http://www.comie.org.mx/congreso/ memoriaelectronica/v10/pdf/area tematica 15/ponencias/1204-F.pdf

Meza, M., Vázquez, M. A. \&, Cordero, G. (2011). Las funciones del asesor técnico pedagógico. Análisis de la documentación oficial. Memoria Académica del Primer Congreso Latinoamericano de Ciencias de la Educación. Mexicali.

Nahmad, S. (Coord.). (2009). Resumen ejecutivo del informe final de la Evaluación cualitativa complementaria del programa Asesor Técnico Pedagógico. México: Centro de Investigaciones y Estudios Superiores en Antropología Social. Recuperado de: http://sep.gob.mx/work/models/sep1/Resource/321/1/images/ resum_ejecutivo.pdf

Rodríguez, M. M. (1996). El asesoramiento en educación. Madrid: Aljibe.

Romero, C. A. (2006). Funciones de asesoramiento escolar en los procesos de cambio en la sociedad del conocimiento: un estudio de caso en las escuelas secundarias de Buenos Aires (Tesis doctoral). Recuperada de http://biblioteca.ucm.es/tesis/ edu/ucm-t29346.pdf

Sánchez, R., Cordero, G., \& Bocanegra, N. (septiembre, 2009) El asesor de zona escolar de educación primaria Tijuana. Percepciones y contradicciones en su función. Memorias electrónicas del X Congreso de Investigación Educativa. Veracruz, México. Recuperado de http://www.comie.org.mx/congreso/memoriaelectronica/v10/pdf/area tematica_16/ponencias/1093-F.pdf 
Funciones y ámbitos de los asesores académicos

para la diversidad lingüística y cultural

María Guadalupe Tinajero Villavicencio

Schmelkes, S. (2004). La política de la educación bilingüe bicultural. En I. Hernaiz (Org.), Educación para la diversidad. Experiencias y desafíos en la educación intercultural bilingüe (pp. 185-196). Buenos Aires: Instituto Internacional de Planeamiento de la Educación/ UNESCO.

Schmelkes, S. (2010). Indígenas rurales, migrantes, urbanos: una educación equivocada, otra educación posible. Pensamiento Iberoamericano, 7, 203-222. Recuperado de http://www.red-redial.net/revista-pensamiento,iberoamericano183-2010-0-7.html

Secretaría de Educación Pública (2007). Programa Sectorial de Educación, 2007-2012. México: Autor.

Secretaría de Educación Pública. (1988). Programa Nacional para la Modernización de la Educación de la educación indígena, 1990-1994. México: Autor.

Secretaría de Educación Pública. (1995). Programa de Desarrollo Educativo, 1995-20oo. México: Autor.

Secretaría de Educación Pública. (2001). Programa Nacional de Educación, 2001-2006. México: Autor.

Secretaría de Educación Pública. (2010). Acuerdo 553 por el que se emiten las Reglas de operación del programa Asesor Técnico Pedagógico y para la atención educativa a la diversidad social, lingüistica y cultural. México: Diario Oficial de la Federación. Recuperado de http://dof.gob.mx/nota_detalle.php?codigo $=5173018 \&$ fecha $=29 / 12 / 2010$

Secretaria de Educación Pública. (2011a). Acuerdo Número 592 por el que se establece la articulación de la educación básica. Recuperado de http://basica.sep.gob.mx/ reformasecundaria/doc/sustento/Acuerdo_592_completo.pdf

Secretaría de Educación Pública. (2011b). Acuerdo 606 por el que se emiten las Reglas de operación del programa Asesor Técnico Pedagógico y para la atención educativa a la diversidad social, lingüistica y cultural. México: Diario Oficial de la Federación. Recuperado en http://www.sep.gob.mx/work/models/sep1/ Resource/9721849d-666e-48b7-8433-oeec1247f1ab/a606.pdf

Secretaría de Educación Pública/Dirección General de Educación Indígena. (1999). Lineamientos generales para la educación intercultural bilingüe. México: Autor.

Secretaría de Educación Pública/Dirección General de Educación Indígena. (2008). Lengua indígena. Parámetros curriculares. Documento curricular para la elaboración de los programas de estudio de las lenguas indígenas. México: Autor.

Secretaría de Educación Pública/Dirección General de Educación Indígena. (2010). Bases generales para la función asesora. Perfil del ATP de Educación Indígena. México: Autor. 
María Guadalupe Tinajero Villavicencio

Secretaría de Educación Pública/Dirección General de Educación Indígena. (2011). Estadística total de educación indígena. Inicial y Básica. Inicio de ciclo escolar 2009-2010. Recuperado de http://basica.sep.gob.mx/dgei/pdf/inicio/informacion/estadisticasDGEIog10.pdf

Tapia, G. (2008). Formación para el asesoramiento a las escuelas: un proceso emergente en México. Profesorado, Revista Electrónica de Currículum y Formación del Profesorado, 12(1). Recuperado de http://www.ugr.es/ recfpro/rev121COL6.pdf

Tójar, H. C. (2006). La investigación cualitativa: comprender y actuar. Madrid: La Muralla.

Universidad Pedagógica Nacional. (2007). Evaluación de diseño, consistencia y resultados (Reporte Técnico). México: Autor. Recuperado de http://www.sep.gob. $\mathrm{mx} /$ work/models/sep1/Resource/120816/1/informe_definitivo.pdf 